Revue des sciences de l'éducation

Du sujet foucaldien au sujet postmoderne. Laïcité et déni du politique dans le système scolaire français

The concept of subject from " foucaldian " to post-modern views : laicism and denial of policies in the French educational system Del sujeto foucaultino al sujeto posmoderno. Laicidad y
negación del carácter político en el sistema escolar francés

Béatrice Mabilon-Bonfils

Volume 34, numéro 2, 2008

Écoles et familles de minorités ethnoculturelles

URI : https://id.erudit.org/iderudit/019690ar

DOI : https://doi.org/10.7202/019690ar

Aller au sommaire du numéro

Éditeur(s)

Revue des sciences de l'éducation

ISSN

0318-479X (imprimé)

1705-0065 (numérique)

Découvrir la revue

Citer cet article

Mabilon-Bonfils, B. (2008). Du sujet foucaldien au sujet postmoderne. Laïcité et déni du politique dans le système scolaire français. Revue des sciences de

l'éducation, 34(2), 465-484. https://doi.org/10.7202/019690ar
Résumé de l'article

Le passage des sociétés modernes aux sociétés postmodernes impose la réévaluation de la notion de sujet et des modalités de construction de la règle scolaire. Si, à l'école, le contrôle devient régulation, la question de la nécessaire intériorisation de la loi scolaire gomme celle de l'arbitraire et de la plurivocité des règles. La spécificité du système éducatif français tient à la construction historique républicaine de la laïcité, abusivement saisie comme neutralité politique ou religieuse. Le déni du caractère négociable et interprétatif des normes scolaires scelle le caractère politique de l'institution scolaire et les difficultés à penser collectivement le lien/lieu scolaire.
Tous droits réservés @ Revue des sciences de l'éducation, 2008
Ce document est protégé par la loi sur le droit d'auteur. L'utilisation des services d'Érudit (y compris la reproduction) est assujettie à sa politique d'utilisation que vous pouvez consulter en ligne.

https://apropos.erudit.org/fr/usagers/politique-dutilisation/ 


\title{
Du sujet foucaldien au sujet postmoderne. Laïcité et déni du politique dans le système scolaire français
}

\author{
Béatrice Mabilon-Bonfils, chercheure associée
}

IREDU-CNRS de Dijon

\begin{abstract}
RÉSUMÉ - Le passage des sociétés modernes aux sociétés postmodernes impose la réévaluation de la notion de sujet et des modalités de construction de la règle scolaire. Si, à l'école, le contrôle devient régulation, la question de la nécessaire intériorisation de la loi scolaire gomme celle de l'arbitraire et de la plurivocité des règles. La spécificité du système éducatif français tient à la construction historique républicaine de la laïcité, abusivement saisie comme neutralité politique ou religieuse. Le déni du caractère négociable et interprétatif des normes scolaires scelle le caractère politique de l'institution scolaire et les difficultés à penser collectivement le lien/lieu scolaire.
\end{abstract}

MOTS CLÉS • école, laïcité, règles et normes, pouvoir politique, représentations.

\section{Introduction}

Les crises sporadiques qui agitent le système scolaire français depuis une vingtaine d'années sont bien plus que la traduction d'un désenchantement de l'école. Elles ne se résument pas non plus à un malaise enseignant, lycéen ou étudiant ni même des jeunes de banlieue. Elles sont à saisir comme symptôme d'une crise de citoyenneté collective, d'une véritable crise du politique, des institutions et, plus largement, de l'intégration républicaine. Or, l'école est bien un lieu politique, tant parce qu'il est le lieu de la citoyenneté que parce que s'y jouent des jeux et enjeux de pouvoir. Cette crise de sens de l'école tient à la remise en cause du consensus dont elle faisait auparavant l'objet, consensus collectif bien que produit hégémonique, dont l'école était le garant et le ferment. Celui-ci reposait sur l'inculcation d'une république moniste par l'école, dans un schéma porté par la laïcité et la méritocratie comme mythes fondateurs et par un contrôle social produisant un sujet discipliné.

Ces remises en cause s'actualisent aujourd'hui au cœur même du système scolaire. Le sens de l'expérience scolaire, dans la double acception de direction et de signification, est en crise; ou bien plutôt, la définition sociale univoque de l'expérience scolaire a vécu, car l'idée même de sujet a changé. La démocratisation de l'enseignement a été une idée neuve, croisée du mouvement de massification de l'école et d'un moment de nécessaire gestion du pluriel. Elle doit aujourd'hui être comprise comme rupture du paradigme fondateur élitiste de l'école de la 
République par l'éclatement des formes identitaires d'appartenance à l'école. Fondateur de la République française, l'idéal de laïcité est devenu prétexte à oblitérer toute prise en compte de la dimension politique de l'institution scolaire et ce, à un moment où le sujet foucaldien ${ }^{1}$ laissait place à un sujet postmoderne. Cela s'est traduit par des difficultés croissantes vécues par l'institution scolaire dans sa gestion de la pluralité et du multiple.

Or, chaque époque construit les sciences sociales qui l'interprètent; mais ces analyses scientifiques participent elles-mêmes rétroactivement à engendrer des mutations culturelles. L'activité scientifique, si elle répond au projet cartésien de maîtrise de la nature, est aussi production sociale. Ainsi, le projet de connaissance scientifique de la société peut aussi se comprendre dans ses prémisses, comme discours de légitimation du procès moderniste de la société, dans une configuration de crise, croisée d'un moment de rupture avec la tradition et d'un mouvement de dynamique de la modernité rationaliste et individualiste.

Aux $19^{\mathrm{e}}$ et $20^{\mathrm{e}}$ siècles, les premiers sociologues, de Durkheim à Weber, de Simmel à Comte ou Marx, ont été des penseurs/panseurs sociaux de la modernité et par delà leurs différences, toutes les sociologies classiques, des sociologies de l'intégration aux sociologies critiques ou aux sociologies utilitaristes, partagent cette même prémisse: il y a correspondance sur des modalités (certes différentes, selon les théories), entre logiques sociales, pratiques et conduites individuelles. L'interprétation univoque du monde social des sociologies naissantes repose sur l'idée que l'inscription sociale des individus les constitue comme sujets, assujettis, soumis au contrôle social, aliénés par le contrôle social, produits du contrôle social. Oscillant entre pôle compréhensif et pôle explicatif, les sociologies naissantes questionnent l'emprise du social sur des individus réifiés: la raison individuelle y est soumise aux exigences du collectif. Il est probable que l'inscription dans le champ des sciences de la réflexion sur le social a nécessité, dans un premier temps, cette dissolution du sujet, que les mutations culturelles liées à la postmodernité remettent aujourd'hui en question. Cependant, dans une sorte d'effet de mode, de retour du balancier, l'engouement des sociologues pour la question du sujet doit être, à notre sens, réévalué; que celui-ci soit appréhendé comme idiot rationnel, ou réduit aux jeux de réalité qu'il construit par le langage réinterprétant le monde où il vit. Développée par Sen (1993), cette expression sociologique renvoie à une critique de l'individualisme méthodologique : la recherche égoïstement rationnelle, par chacun, de son propre intérêt, peut éloigner tout le monde de la solution collective la meilleure. Il en découle la nécessité de tenir compte de l'engagement comme composante du comportement. En effet: L'homme purement économique est à vrai dire un demeuré social. La théorie économique s'est beaucoup occupée de cet idiot rationnel, drapé dans la gloire de son classement de préférences, unique et multifonctionnel (1993, p. 110).

Le présent article vise à appréhender ces mutations dans un cadre théorique renouvelé, permettant d'interpréter les relations dialogiques autant que combinées 
entre rapports sociaux et expérience subjective des individus. Par relations dialogiques, nous entendons qui fonctionnent par opposition et dialoguent par combinaison; donc qui coexistent, selon la pensée de Morin, dans tous ses ouvrages. Longtemps négligée, voire niée, sous l'hégémonie de système d'actions collectives, la capacité réflexive des individus resurgit, et il faut trouver un entre-deux, c'està-dire une situation intermédiaire qui articule ces conceptions, apparemment opposées, entre un sujet-reflet du système social et un sujet social menacé de dissolution.

Une sociologie du sujet renversant cette opposition mutilante de la complexité du réel s'impose: entre un objet trop plein de sens et un sujet en quête de sens. Les sociologues seront alors en mesure d'appréhender que ce qui a statut de réalité pour les sujets constitue des territoires virtuels et réels à la fois. Il s'agit de prendre en compte que la réalité est structurée par les représentations qui la construisent. Dans cette herméneutique ${ }^{2}$ du sujet, nous plaidons pour une appréhension endogène des activités sociales, qui ne se réduise pas de manière positiviste à une ethnométhodologie. Les sociologues qui adoptent cette démarche (développée aux États-Unis, depuis les années 1960), centrent leur intérêt sur le savoir ordinaire des membres d'une société, et nient la rupture entre le savoir savant/celui des sociologues, et le savoir pratique des individus.

Ainsi, le passage des sociétés modernes aux sociétés postmodernes impose la réévaluation de la notion de sujet, ce qui ne peut être sans effet sur la raison sociologique. Or, si dans nos sociétés, le contrôle devient régulation, l'école est au cœur de ces mutations en tant qu'elle participe au modelage social de l'individu, à la construction sociale des sujets; mais de quels sujets?

Il s'agira alors d'aborder cette nécessité d'une rupture de paradigme, du sujet foucaldien à un sujet postmoderne (voir partie I du présent article). La spécificité du système éducatif français impose un détour historique: dans notre république moniste qui s'oppose au pluralisme, où l'Autre doit devenir le même, la construction républicaine de la laïcité s'articule autour du déni du politique dans le système éducatif français, constitutif de cette négation du sujet (voir partie II). Le déni du caractère négociable et interprétatif des normes scolaires scelle à la fois le caractère politique de l'institution scolaire et les difficultés à penser collectivement le lien/lieu dans un arbitraire de la règle à la fois nécessaire et non assumé (voir partie III).

\section{Du sujet foucaldien au sujet postmoderne: une rupture de paradigme}

Si la notion même de sujet est centrale pour comprendre ce qui se joue dans les institutions en général et dans l'école en particulier, c'est l'ambivalence même de l'idée de sujet qui est féconde: être sujet, est-ce être assujetti? Ou bien devenir sujet-acteur de ses choix? La rupture de paradigme que connaît notre système sociétal tient largement à ce questionnement. 


\subsection{L'école, lieu de définition du sujet discipliné}

L'école est un instrument idéologique d'État, en ce que tout État joue sa pérennité dans la formation du citoyen. La contribution de l'État moderne à la naissance du citoyen renvoie à un processus de civilisation des mours, selon l'expression d'Elias $(1939,2002)$, de refoulement des sensibilités et des émotions. Ce processus s'accompagne d'un sentiment d'obligation et d'un système d'autocontraintes intériorisées par la socialisation que l'école participe à construire. Or, dans l'État-Nation à la française, le sujet discipliné en est l'aboutissement. Foucault (1975) utilise le concept de gouvernementalité, entendu comme l'ensemble constitué par les institutions et les procédures qui permettent d'exercer cette forme bien spécifique de pouvoir. Celui-ci a pour cible l'ensemble de la population; pour forme majeure de savoir, l'économie politique; pour instrument technique, les dispositifs de sécurité; pour enjeu politique, les hommes dans leurs rapports avec les manières de penser, de faire, de sentir, les corps et les esprits.

L'école, agent de gouvernementalité, est un lieu de production de ce citoyen discipliné par la microphysique du pouvoir qu'elle institue. Celle-ci suppose que le pouvoir, qui s'exerce dans une institution, est conçu comme une stratégie; que ses effets de domination ne sont pas attribués à une appropriation, mais à des dispositions, à des manœuvres, à des tactiques, à des techniques, à des fonctionnements. Ce pouvoir ne s'applique pas, purement et simplement, comme une obligation ou une interdiction, à ceux qui ne l'ont pas: il les investit, passe par eux et à travers eux (Foucault, 1975). Si le pouvoir investit le tissu même de l'école, on ne saurait l'entendre uniquement comme sanction et répression. Il circule et fonctionne en chaine, et c'est ce fonctionnement des rituels politiques du pouvoir qui instaure des relations mobiles, asymétriques et inégalitaires. Ces relations se déploient dans la mise en œuvre quotidienne de technologies politiques, dans une localisation spatiale et temporelle. La volonté de pouvoir de l'école s'applique à l'élève et à l'enseignant: ce sont des individus à connaître et à classer, soumis qu'ils sont au code scolaire des conduites et des performances, qui marque les moments d'un premier formatage de l'individuel, par ces relations de pouvoir et leur maillage. Technique de pouvoir et procédure de savoir se nourrissent mutuellement.

Le pouvoir passe alors par la reconnaissance de l'individu comme objet de savoir: entre techniques de savoir et stratégies de pouvoir, nulle extériorité, nous apprend la thèse foucaldienne. En effet, selon Foucault, le pouvoir n'est pas extérieur aux individus, mais fait corps avec eux et s'exprime dans les relations et interactions qu'ils construisent au quotidien. Le contrôle de l'activité de l'ensemble des acteurs de l'établissement scolaire passe autant par la gestion de l'espace que par le contrôle du temps. Toute une discipline de vie, du temps et des énergies est mise en place. Selon la localisation des individus, autour de la discipline s'organise un espace analytique par un principe de quadrillage ou de localisation élémentaire, où chaque individu doit trouver sa place et chaque emplacement un individu. L'architecture même de l'école implique l'organisation d'espaces complexes, à la 
fois architecturaux, fonctionnels et hiérarchiques. Tout l'espace est ainsi codé selon des emplacements fonctionnels qui assurent la fixation des élèves, permettent leur circulation, marquent les places et les valeurs, tout en décrivant une série finie de relations possibles entre élèves, entre enseignants et entre élèves et enseignants. Lieu clos, l'établissement scolaire, citadelle fortifiée dont les entrées et sorties sont réglementées, est organisé autour d'un espace vide, la cour de récréation placée au centre des bâtiments. À la fois lieu de circulation et de surveillance, l'école est soumise au regard panoptique ${ }^{3}$, tout en étant investie physiquement et affectivement par les élèves. De plus, l'espace disciplinaire tend à se diviser en autant de parcelles qu'il y a de corps et d'éléments à répartir, pour éviter les distributions par groupes. Il faut annuler les répartitions indécises, la disparition incontrôlée des individus, leur circulation diffuse, leur coagulation. C'est pourquoi la gestion de l'occupation des salles (planning) et le contrôle des présences sont les instruments par excellence de la régulation scolaire. Il s'agit d'établir les présences et les absences, de savoir où et comment retrouver les individus, d'instaurer les communications utiles, d'interrompre les autres, de pouvoir à chaque instant surveiller la conduite de chacun, l'apprécier, la sanctionner, en mesurer les qualités ou les mérites. Procédure donc, pour connaître, pour maîtriser et pour utiliser.

Par la mise en rang se disposent les éléments soumis au système de contrôle et de régulation. Dès lors, les sujets disciplinés deviennent interchangeables, puisque chacun se définit par la place qu'il occupe dans une série et par l'écart qui le sépare des autres. La note attribuée aux élèves et la note administrative et pédagogique affectée aux enseignants participent de la même logique: elles s'articulent à des procédures de négociation et à des processus de pouvoir. Au rang qu'occupe chaque élève dans la hiérarchie des élèves (dont les variables instituées dépassent les seules performances scolaires), correspond grosso modo le rang qu'occupe chaque enseignant dans la hiérarchie professionnelle ou statutaire du groupe des enseignants. La discipline, à la fois art du rang et technique de transformation des arrangements, individualise les corps par une localisation qui ne les implante pas, mais les distribue et les fait circuler dans un réseau de relations. La règle des emplacements fonctionnels va peu à peu, dans les institutions disciplinaires, coder un espace que l'architecture laissait en général disponible et prêt à plusieurs usages...

Ainsi, le rang fait partie des déterminants de la vie scolaire proprement dite: rangées d'élèves dans la classe, les couloirs, la cour, rang d'appel, rang attributif (chef de classe), rang d'épreuves, alignement des classes d'âge, succession des matières enseignées dans une graduation des difficultés. Le rang est en fait le marqueur d'une hiérarchie du savoir et des capacités, des valeurs et des mérites.

Par ailleurs, l'encadrement et le contrôle des activités passent par un minutage et une mise en ordre des emplois du temps. Le régime des sanctions (positives ou négatives) sert à contraindre les individus à des activités déterminées tout en réglant des cycles de répétitions. La discipline se manifeste par la régularité de la vie scolaire et la gestion relativement rigoureuse du temps scolaire. L'emploi du 
temps opère un quadrillage serré des activités, rythmées par des obligations. Une telle régulation vise à garantir un temps intégralement utile.

Il y a aussi élaboration temporelle de l'acte dans une sorte de schéma anatomochronologique du comportement, selon l'expression de Foucault (1975). Toute activité pédagogique est décomposable en ses éléments. Position du corps et position des membres sont autant de techniques d'assujettissement du corps par incorporation de la temporalité et contrôles minutieux du pouvoir. Le modèle disciplinaire organise une nouvelle économie du temps d'apprentissage.

En devenant cible pour les mécanismes de pouvoir, le corps s'offre à de nouvelles formes de savoir. Le corps manipulé par l'autorité devient corps de l'exercice, corps du dressage utile, dans lequel s'annoncent des contraintes de nature fonctionnelle.

Ainsi, parmi les compétences qu'enseignants et élèves doivent acquérir, certaines tiennent à la maîtrise du corps (occupation de l'espace, distance à autrui, maintien, mouvements normalisés) et d'autres à la gestion des émotions (ni colère, ni familiarité).

L'apprentissage du métier d'élève, tout comme du rôle d'enseignant, ne peut être réduit à l'acquisition de savoirs et de savoir-faire, mais repose sur la définition d'un savoir-être légitime, requis par l'institution. Comme les corps, l'espace est analysé par les disciplines et techniques du pouvoir qui décomposent et recomposent les activités.

La division du temps s'applique aux corps. Elle a aussi pour fonction d'assurer que le sujet discipliné atteigne le niveau statutaire attendu, et de garantir que son apprentissage et celui des autres sont uniformes. Il revient à l'exercice (du corps et de l'esprit), de jouer dans le sens d'un assujettissement inachevé de l'individu, à la fois victime et producteur de violence. Par l'application d'une discipline, l'exercice agit comme élément dans une technologie politique du corps et de la durée.

Or, nos sociétés postmodernes connaissent des mutations, dans la conception même de l'assujettissement, qui ne sont pas sans affecter cette mise en discipline des sujets.

\subsection{Du sujet discipliné au sujet postmoderne}

Qu'il s'agisse des représentations sociales de la réussite individuelle dans une société de marché, de l'idéologie de la performance, du narcissisme consumériste, de l'idéologie individualiste du marché, l'entrée de nos sociétés dans la phase postindustrielle de leur histoire se caractérise par une émergence, à tout le moins un renforcement des comportements ou des idéologies individualistes. Sur le plan théorique, l'horizon normatif des années 1980 se définit, à l'encontre des structuralismes et des déconstructivismes des décennies précédentes, par un parti-pris nouveau dans les sciences sociales: celui du caractère indépassable de l'humanisme, voire de l'humanité du sujet. Ainsi, dans le champ de la pratique sociale comme 
dans celui des problématiques théoriques, la subjectivation du rapport social semble naître de ce postmodernisme. De Munck (1997) explique que tout se passe comme si l'Occident entrait dans une nouvelle période alexandrine, où se cultivent le souci de soi et le salut individuel, dans un monde qu'on a renoncé à maitriser sur le plan collectif. Aucune théorie sociologique ne peut faire l'économie d'une inscription du sujet dans la concrétude des rapports sociaux et historiques. Foucault (1975) montre d'ailleurs en quoi une histoire des idées et des valeurs, non ancrée sur les rapports sociaux et les rapports de pouvoirs, est vide. Il nous faut aujourd'hui penser une théorie du sujet en dialogue et en dépassement avec l'approche de Foucault. Précisons en quelques mots comment cette approche foucaldienne du pouvoir et du sujet, appliquée à l'école et déjà explicitée par Mabilon-Bonfils et Saadoun (2001), peut aujourd'hui orienter de nouveaux modes d'investigation.

Foucault lie la construction moderne du sujet à des dispositifs de savoir/pouvoir de type disciplinaire. Il fait l'hypothèse que l'émergence effective du sujet passe par l'instauration d'un modèle de normativité, de contrôle panoptique orienté et par des techniques de pouvoirs, comme nous l'avons précédemment explicité. Le propre de la raison moderne est bien de construire un rapport formaliste à la norme, en évacuant au maximum les marges d'indétermination par un quadrillage strict introduisant la règle dans les moindres interstices du social. Ce modèle se construit dans une passion de l'unité, qui est celle de la raison et de l'État, et dont l'école est un des acteurs. La norme doit être unique, sans lacune, sans concurrence possible. Si le pouvoir s'exprime de façon plurielle, dans l'école (comme d'ailleurs dans la prison ou l'asile), cette dissémination ne conduit pas à penser la pluralité des mondes normatifs. Ce sont les convergences formelles qui orientent le modèle foucaldien du pouvoir. Le contrôle hiérarchique organise un espace de visibilité sociale tout en cherchant à rester invisible. Ce dispositif de contrôle est porteur d'objectivation mais aussi de subjectivation. L'objectivation cognitive et pratique des individus est l'effet du dispositif de surveillance, mais le contrôle ne fonctionne que parce qu'il est intériorisé par le sujet: en d'autres termes, la figure du sujet est nécessaire au dispositif disciplinaire, à la fois condition de possibilité et effet du dispositif, mais seul le sujet maîtrisé/contrôlé est pris en compte.

Depuis une vingtaine d'années dans nos sociétés postmodernes, il y a rupture de ce mode de régulation: ce n'est pas que la rationalisation et le contrôle disparaissent, mais ils se poursuivent sous d'autres formes, encore incertaines, plus fluides et moins facilement objectivables. Dans ces conditions, comment appréhender les institutions comme cadre de ces processus? Si le modèle de normativité change, c'est que la norme se fait plus indéterminée, interprétée, voire réinterprétée en situation. Pour une même situation, coexistent une pluralité de normes, de modes de justification et de ressources. L'école est soumise à de nombreux codes normatifs, pas toujours congruents entre eux, des exigences civiques à celles du marché, des exigences de performance individuelle au respect des identités collectives et indi- 
viduelles. La complexité et la plurivocité de règles s'opposent ainsi à la complétude et au caractère univoque de la règle qui définissaient le modèle disciplinaire. Les modalités mêmes du contrôle changent. Même s'il est disséminé, on passe d'un contrôle centralisé à un contrôle local, latéral, à côté, plus horizontal, ce en quoi d'ailleurs la visée propre au pouvoir postmoderne - se rendre plus invisible encore -, est renforcée par ce mode de contrôle social. La coercition et le symbole, instruments du pouvoir, gardent leur efficacité, mais d'une nouvelle façon: le contrôle contemporain porte globalement sur des compétences informelles. Il ne vise plus tant la normalisation qu'il n'accepte des révisions perpétuelles de modes d'action. Il ne punit plus pour inclure; il exclut pour gouverner l'hétérogénéité, pour gérer l'incertitude. Ainsi s'interprètent les règles dans le lieu scolaire, qui requièrent la mobilité des élèves entre filières et établissements, qui requièrent les modes de sélection et de bannissement dans des filières ou des établissements, ce qui génère d'ailleurs un sentiment d'abandon chez certains élèves. Les processus d'inclusion/ exclusion prennent le relais des processus de surveillance/contrôle.

Certes, la pensée foulcaldienne est réductionniste à certains égards: en effet, la compréhension du sens se ramène à l'explication des discours; les aspects intérieurs de la signification y sont souvent gommés, car identifiés aux pratiques de pouvoir; la raison moderne est réduite à la raison formaliste; enfin, il existe une ontologie indépassable du pouvoir. Toutefois, cette approche, non idéaliste de la raison et de la société, constitue une balise pertinente, au seuil d'une mutation probable de paradigme, selon laquelle aujourd'hui le contrôle social deviendrait procédural, et la normativité plurale.

Sans aucun doute, l'histoire du sujet postmoderne ne peut se réduire à l'unité d'une figure, dans la mesure où les pratiques du souci de soi sont à renvoyer à une pluralité de figures du sujet, pris dans la subversion des codes, la dispersion des autorités et des sources de légitimité, la pluralité des mécanismes institutionnels et l'autonomie instituée.

Ce détour nécessaire par l'idée de sujet plaide, selon nous, pour l'introduction d'une dimension politique dans cette théorie du sujet en gestation dans les sciences sociales, car les pratiques institutionnelles ne sont jamais neutres. Ardoino déplore d'ailleurs cet [...] oubli du politique au cœur de la question éducative [...] et appelle de ses vœux la réhabilitation de la conscience critique et de la place légitime du projet politique dans les pratiques éducatives (1985, p. 6).

Un regard de type politologique s'impose pour comprendre comment, dans le système politique français, l'idée de laïcité a connu un infléchissement terminologique notable et non anodin, porteur de cette dissolution du sujet.

\section{La laïcité et le déni du politique dans le système éducatif français: une république moniste}

Contrairement à ce que véhiculent nos idéologies françaises centrales, l'espace public et l'espace privé ne sont pas étanches. La laïcité, corrélée au projet mérito- 
cratique, a été l'instrument d'une construction politique de notre citoyenneté. Il y a un lien historique, voire nécessaire, entre république et laïcité, mais celle-ci est abusivement saisie comme neutralité politique (autant que religieuse), ce qui engendre un véritable déni du politique dans le lieu scolaire.

\subsection{La République française et la laïcité: un lien nécessaire}

$\mathrm{Si}$, dans la définition du citoyen, l'État éducateur joue sa réalisation et sa pérennité, c'est bien dans un processus de contractualisation social et politique de l'éducation d'une nation unifiée que se conçoit l'histoire politique de l'école en France. Cette histoire politique doit donc être purifiée des scories des histoires singulières. L'école institue et légitime tour à tour une vision eschatologique ${ }^{4}$ de la société française, par laquelle le peuple est rendu pleinement souverain, sous l'éclairage généreux de la Raison, des Lumières et de l'universalité conquérante des savoirs émancipateurs.

En tant que lieu cardinal de la citoyenneté, l'école de la République a historiquement travaillé à la construction unitaire de la citoyenneté française, conçue comme déni des allégeances particulières et comme topos fondateur de neutralisation des lieux et des milieux. Certes, la laïcité n'est pas neutre: c'est un véritable projet politique de combat et une manière de concevoir le lien civil et politique et, plus précisément, le rapport de la forme scolaire aux logiques d'État. C'est bien en ce sens qu'il faut concevoir le projet de sécularisation. Le projet de laïcisation et de sécularisation de l'école, du savoir et plus généralement de la société, témoigne d'une recherche de mainmise de l'État sur les corps et les esprits. Tout au long de l'histoire scolaire, la querelle qui oppose les républicains et l'Église est récurrente, de la loi Guizot déterminant le principe de la liberté de l'enseignement pour le primaire en 1833 à la loi Falloux pour le secondaire en 1850, à la loi Dupanloup pour l'enseignement supérieur en 1875 , jusqu'à ce que la séparation de l'Église et de l'État en modifie les termes, en 1905. À un moment donné de cette histoire, ce paradigme d'un savoir unifié, qui se fonde sur un progrès continu de la connaissance, repose sur la construction d'un ordre scolaire, stratégie la plus efficace de cette diffusion du savoir par l'instauration d'une instruction gratuite et obligatoire, mais surtout résolument laïque. Enseignement des principes de la raison, la laïcité y est pensée comme une variation de l'universalité. Ainsi, l'unité méthodique du savoir renvoie à l'unité de l'esprit humain, dans cette construction d'un espace commun de raison entre maîtres et élèves. La circulaire du ministre de l'Instruction publique du 17 novembre 1884, dite Lettre de Jules Ferry aux instituteurs, établit d'ailleurs une distinction entre deux domaines trop longtemps confondus: celui des croyances, qui sont personnelles, libres et variables, et celui des connaissances, qui sont communes et indispensables à tous, de l'aveu de tous.

La construction d'une citoyenneté totalisante constitue bien une exception française, entrée en crise avec la remise en cause du modèle de la République moniste. L'idée de citoyenneté nationale fondée sur le contrat, héritière des théories d'inspiration rousseauiste du pacte social volontariste, inscrit le sujet-citoyen 
dans une appartenance collective, perpétuation des liens sacrés des individus au tout, où tradition nationale et contrat social sont complémentaires. Dans sa tradition jacobine, l'exception française commande à la fois l'allégeance des citoyens à la Cité et subordonne cette soumission au culte de la raison universelle. Ce faisant, seul le citoyen est sujet de droit: citoyen abstrait dans l'unité abstraite qu'incarne la République. Unifiée et centralisée plus tôt que la plupart des autres États européens, la France a été plus radicalement sécularisée et laïcisée et a développé des concepts novateurs comme les Droits de l'homme et du citoyen, paradoxalement liés à l'équation État = nation = peuple, faisant de la loi un paradigme universel. Le citoyen y est l'homme d'un seul État, d'une seule foi : le républicanisme laïque et égalitaire; d'une seule idéologie: les droits de l'homme. Selon Étienne, Giordan et Lafont (1999), le citoyen abstrait n'a ni âge, ni sexe, ni origine sociale, ni origine ethnique, alors que la Révolution est à la fois française et bourgeoise. Ce que l'universalité des valeurs de la bourgeoisie conforte dans l'instant de la création citoyenne, c'est la part unique et nécessaire d'une culture de la raison. L'identification des valeurs bourgeoises aux idéaux nationaux marque cette transcendance du travail de l'unité qui s'affirme résolument contre la diversité. Mue par une philosophie de l'Un, cette citoyenneté réduit l'Autre au Même. La centralité du noyau citoyen tient à ce qu'elle se construit, comme mode d'appartenance, en rupture avec les autres formes d'allégeance, qu'elles soient locales, parochiales ${ }^{5}$, claniques. La citoyenneté relègue les solidarités mécaniques à la sphère du culturel et non du politique, à la sphère de l'intimité et non du public. Ce concept de solidarités mécaniques a été forgé par Durkheim (1893), pour définir le lien social des sociétés traditionnelles, fondé sur la similitude et l'indifférenciation sociales.

En tant que déni des allégeances particulières, qu'elles soient infra ou supranationales, d'une part, ou économiques et sociales, d'autre part, la citoyenneté est alors ce projet politique fondateur, vécu comme forme quasi naturalisée d'appartenance qui construit un sujet politique unidimensionnel.

Si le projet fondateur de l'école républicaine est l'idée révolutionnaire d'une acculturation des individus par l'école, lieu de civilisation, encore fallait-il que la sélection scolaire ne soit plus sociale; c'est ainsi que des générations d'instituteurs, porte-parole des Lumières et du positivisme colonisateur, ont intériorisé l'idéal méritocratique: négation des milieux d'appartenance des enfants, l'école devenait ce lieu qui les neutralisait. L'école laïque était bien alors au service de la République. De la négation des milieux, entendus comme matrice des variables sociales, sexuelles, ethniques, régionales, idéologiques et singulières des acteurs de l'école, à la prise en compte partielle et partiale des milieux par le topos scolaire (ou lieu commun), le projet fondateur de l'école de la République est la construction d'un système scolaire, homogène et homogénéisant les différents milieux, dans un lieu de savoir, par delà la concurrence des autres lieux supposés exogènes. La politique scolaire de la Troisième République, fondant l'unité de la Nation autour de l'idéologie républicaine, inaugure l'entrée dans l'ère de l'État éducateur. L'uniformité, 
par la négation des milieux qu'assure la centralisation, doit renforcer l'unité nationale. Ainsi, l'école reçoit la fonction d'abolir les particularismes, notamment locaux, que ce soit par l'imposition du français face aux patois encore usuels dans la vie courante, par l'imposition du système métrique face aux mesures locales (corde, pouce, toise), du franc comme mesure des prix face aux louis et aux écus, ou encore par le refus d'un enseignement de l'histoire ou de la géographie locales, afin de modeler une conscience collective nationale par delà les résistances. L'école, et particulièrement l'école du village, gratuite et obligatoire, a organisé le processus d'acculturation finale qui a transformé les Français en Français, qui finalement les a civilisés. Pour Weber (1983) dans La fin des terroirs, l'école républicaine, insérée dans un processus de civilisation cohérent, parvient ainsi à maîtriser le travail de subjectivation populaire par la construction de ce sujet social à la fois abstrait et universel, conforme à l'idéal de la science positiviste et de la citoyenneté républicaine. Dès lors, l'inculcation des valeurs citoyennes et d'une idée abstraite de l'homme est compensée par le sentiment concret d'appartenance nationale (le petit Breton perdait sa langue, mais devenait patriote). L'école républicaine a ainsi été, à la fois, une entreprise d'alphabétisation et d'égalisation des conditions des connaissances dans sa logique méritocratique, mais aussi un laminoir des différences culturelles et linguistiques. La francisation républicaine, qui a été essentiellement l'œuvre de l'école, a été une nationalisation, une intériorisation de l'État-Nation et de son histoire francophone, parisienne, monocentrée, ancrée dans l'immémorial Gaulois. À ce projet séculier s'ajoute progressivement une volonté d'unification du cursus scolaire et, notamment, d'unité du primaire et du secondaire.

CEuvre de compromis, la laïcité correspond donc à un mode radicalement nouveau d'institution du social et du politique, à la fois résultat d'une histoire singulière, solidaire de l'histoire républicaine et porteuse de la volonté de soustraire les institutions et particulièrement l'école au pouvoir des clercs, dans un contexte politique de combat pour la liberté de l'enseignement. La laïcité tire son origine comme son originalité de la tradition française de contestation du pouvoir clérical. Cependant, dans un contexte socioculturel d'émergence du sujet post-moderne, les modalités de la laïcité doivent être réévaluées à la lumière des mutations des publics notamment scolaires. Par son fonctionnement, en apparence, uniforme, par la construction légitimée et légitimante de l'excellence scolaire, l'école construit une représentation officielle de la valeur scolaire des élèves par delà leurs milieux d'appartenance

\subsection{L'idéologie laïque et le déni du politique}

L'idéologie de ce système global, au sens de perspective sous laquelle le sujet se représente les valeurs, les normes, les fins posées par sa société, était la laïcité et ses dérives, le laïcisme. Comme le souligne Coq: Parce que l'immanence républicaine de l'homme est un mythe, parce que la société républicaine est fragile et que la laïcité 
est une valeur à part entière, l'école républicaine a un rôle fondamental à jouer dans la transmission des valeurs fondamentales qui l'animent et particulièrement la laïcité (1995, p. 128), ce qui semble sceller le caractère politique de toute institution socialisante et spécifiquement l'école. Le laïcisme figure au cœur de la concurrence qui a opposé, au $19^{\mathrm{e}}$ siècle, les mondes religieux et politique quant au monopole du discours de légitimité sur le monde. Dans ce contexte, le laïcisme a été à la République ce que le scientisme a été à la science. Collectivement, le modèle de laïcité à la française, qui est pourtant une exception politique, nous semble aller de soi. Tout se passe comme si la laïcité s'était imposée comme une évidence, d'autant mieux partagée que chacun la définit à sa manière (Gautherin, 2000). Or, la laïcité, œuvre de compromis de la III ${ }^{e}$ République, n'est pas tant une articulation des activités privées et publiques à l'école, une éviction des religions dans l'école, une neutralité religieuse ou d'opinion revendiquée, qu'un véritable modèle politique d'imposition et de légitimation d'un ordre social supposé pacifié, et conçu comme emblème de la conception républicaine de l'espace public. Ce modèle se confond partiellement avec la conception républicaine qui, dans l'espace public et politique, fait prévaloir l'intérêt général sur les intérêts particuliers, universalisme né des philosophies rousseauistes des Lumières. Aujourd'hui, la laïcité renvoie plutôt à une anti-idéologie (dans la mesure où elle est présentée comme une garantie d'objectivité de l'enseignement dispensé), même si cela ne va pas sans occasionner des effets pervers: sous couvert de respect des opinions, d'apolitisme, la laïcité est devenue un prétexte pour oblitérer toute prise en compte explicite des valeurs. En gommant toutes les caractéristiques relevant des champs religieux et politique, l'idée de laïcité conduirait, au nom d'une supposée neutralité, à occulter les débats autour d'enjeux sociaux ou politiques. La dimension politique semble curieusement politiquement incorrecte aux yeux des acteurs du monde scolaire. Ils refusent d'admettre que l'école puisse être un lieu politique et, par conséquent, qu'un regard politologique puisse être jeté sur son fonctionnement. C'est justement le défi à relever pour le sociologue: comprendre les implications de cette réticence collective à penser l'école comme une cité politique, y compris - et peut-être surtout - si elle peut être pensée parfois comme la Cité idéale.

Ce modèle est aujourd'hui fondé sur la revendication d'une neutralité politique de l'école. Les représentations sociales ont connu un glissement sémantique qui s'est opéré en assimilant neutralité, non seulement religieuse mais politique de l'école, voire de l'État, et laïcité. En étiquetant le monde, les représentations sociales orientent les pratiques sociales. La question de la neutralité politique de l'État républicain ne peut pas ne pas être posée. S'il est historiquement anachronique d'assimiler laïcité et neutralité politique, c'est par une méconnaissance du sens originel. En effet, la laïcité, résultat d'un conflit au cours duquel l'État s'est émancipé de l'institution religieuse, n'est pas, dans l'esprit de Ferry et des créateurs de l'école républicaine, synonyme de neutralité politique, mais de neutralité religieuse, même si le modèle fondateur de la laïcité a des fondements quasi-religieux dans 
la sacralisation du savoir (particulièrement du savoir abstrait), supposés gages d'une morale laïque commune à transmettre. C'était donc une idéologie très engagée politiquement, puisqu'il s'agit, pour l'école de la III ${ }^{e}$ République, d'inculquer la Patrie et la République. Il fallait, par l'école, construire la République, dont l'État national est une référence identitaire, voire une valeur ontologique. Dans le contexte de l'État moderne, les schémas moraux de la tradition ont perdu de leur force et la sécurité ontologique, faiblement fondée, s'appuie désormais sur des routines sans signification morale. Selon Giddens (2005), la communauté créée par les symboles nationaux fournit alors cette sécurité ontologique qui fait défaut. En tout état de cause, l'État-Nation doit assurer sa continuité par cet habitus national suscitant ce sentiment national, image d'un nous valorisant. Ce sentiment et cette idéologie de la communauté nationale ont une fonction cruciale dans l'architecture des institutions démocratiques, en ce que la valeur identitaire de l'État-Nation sert à légitimer les institutions politiques. Les institutions d'enseignement public sont donc orientées vers l'approfondissement et le renforcement d'un enseignement du nous presque exclusivement axé sur la tradition nationale, comme le démontre Citron (1989). L'histoire de la mémoire est la question qui se pose à la modernité, autant comme marquage des traces du passé que comme forme symbolique d'identification collective. Parmi les symboles de l'unité nationale que l'école sélectionne dans son travail de ré-interprétation de la mémoire afin de promouvoir une culture de l'Unique, il en est qui consacrent cette capture de l'histoire: quand l'école véhicule d'une façon caricaturale et par le moyen de clichés réducteurs et producteurs de sens la gloire des grands hommes qui ont forgé l'unité de la France, de Vercingétorix à Charlemagne, de Jeanne d'Arc à Louis XIV ou bien encore à Napoléon. La littérature elle-même glorifie ses héros. Victor Hugo et Lamartine deviendront les auteurs emblématiques de l'école de la III République. Les vertus citoyennes acculturantes, qui enseignent amour de la patrie et culte de l'héroïsme charismatique, rejettent à la périphérie, par une sorte d'amnésie, les caractères singuliers des ethnies, des langues et des peuples qui prévalent dans la construction des identités et des communautés. C'est que tout projet d'enseignement, par le fait même qu'il met en relation des individus, qu'il les contraint à vivre des interactions, à respecter des normes, des règles et des pratiques, participe au moins partiellement à l'intériorisation des valeurs centrales d'une société. Ce qui justifie la formule suivante: pour apprendre, il faut accepter de désapprendre; accepter en somme la normalisation et le contrôle de ses propres conduites, représentations et positions. L'école se justifie dans l'exigence d'une citoyenneté éclairée, c'est-à-dire incommode, et ce, dans le cadre même de la constitution des vertus citoyennes - par articulation des savoirs au savoir-faire et au savoir-être. Les récents débats sur la construction partiellement amnésique de la mémoire coloniale française dans et par l'école en attestent. Ce n'est probablement qu'après mai 1968, dans un climat droitier, d'imputation de gauchisme au corps enseignant, d'un fantasme d'endoctrinement politique des masses par l'école, qu'une circulaire du 
ministre Haby (1975) identifiant laïcité et neutralité politique scelle le glissement sémantique. Celui-ci préconise une attitude objective laïque devant les problèmes religieux et politiques. Selon Haby, l'État est chargé, de par la délégation qui lui en est faite, d'assurer une formation dans un champ limité et qui, de tradition, laisse à l'écart tous les domaines controversés des connaissances et des modes de pensée dans lesquels la responsabilité de définir les objectifs éducatifs ne peut être abandonnée à l'école en tant qu'organisme, à son administration, à un ou plusieurs enseignants, voire au groupe d'élèves. Cette inflexion n'est pas anodine; elle participe du déni du politique dans le lieu scolaire, ce qui nous permet alors d'appréhender autrement la question centrale de l'arbitraire de la règle scolaire.

\section{Le déni du politique dans le lieu scolaire et l'arbitraire de la règle}

Comme toute institution, l'école participe au modelage social de l'individu, à la fabrique du sujet, en lui imposant les valeurs centrales de cohésion de sa société. Ainsi, la socialisation est confrontation à l'autre; elle est à la fois apprentissage, inculcation, négociation et imposition. Pour tisser du lien social et donc pour survivre, toute société doit (re)produire sa culture et ses structures sociales par des processus par lesquels les individus intériorisent des valeurs, des principes moraux, idéaux et sociaux, qui permettent de classer les individus, les situations, ou les comportements jugés souhaitables ou non par le groupe. La société ne peut le faire qu'en générant des mécanismes d'institutionnalisation du social, grâce aux processus de socialisation comme éléments canalisateurs de violence. Dès lors, la question de l'intériorisation de la loi scolaire rebondit. C'est bien un défi pour l'école républicaine que de socialiser la jeune génération et de construire une citoyenneté dans un espace d'arbitraire social collectivement intériorisé, mais de moins en moins bien. En effet, les mutations du sujet contemporain, moins discipliné que contrôlé, infléchissent la production des règles scolaires. La définition des règles est toujours le fruit d'un processus politique. Les règles scolaires n'y échappent pas, alors même que le déni scolaire de leur plurivocité relève d'un déni du Politique, que réactive la dénégation collective du caractère violentogène de l'école. Cependant, il faut toujours compter... avec le retour du refoulé. L'arbitraire de la règle est alors à saisir comme aporie.

\subsection{L'intériorisation de la Loi scolaire et la production des règles scolaires}

Pour saisir la production de l'ordre scolaire, non que celui-ci puisse être conçu comme agencement stable et cristallin mais bien comme volute de fumée, ordre instable et mouvant, il nous faut comprendre les mécanismes de production des règles qui président à l'ordonnancement de l'organisation scolaire. Il ne s'agit pas ici de réfléchir aux modalités politiques collectives de définition des règles, mais bien de comprendre comment la puissance souterraine du social est toujours active (Maffesoli, 2002), et ce, même au sein de l'institution scolaire, lieu d'inculcation 
des normes collectives, de socialisation des jeunes générations et de construction de la citoyenneté. Dans l'école, comme dans toute organisation, les règles doivent être interprétées et ce caractère interprétatif est l'un des éléments qui signe le caractère politique de l'école, matérialisé par la plurivocité des règles scolaires. Par exemple, la règle collective de ponctualité, expliquée aux élèves en début d'année généralement par le professeur principal à la lecture du règlement intérieur, fait l'objet d'interprétations plurielles, selon l'enseignant, probablement aussi selon la situation, et parfois même selon l'élève concerné. Si les mots font en partie, les choses, l'observation même de la gestion de la ponctualité par les enseignants montre qu'elle fait l'objet de multiples amendements, improvisations, négociations. Dans les relations de pouvoir qui s'instaurent entre un enseignant et sa classe, ces fluctuations de la règle sont un élément possible de production collective d'un ordre scolaire, fait de désordres temporairement stabilisés, au sein d'une organisation fixant un cadre, qu'il est toujours possible de déborder. L'arbitraire de la règle révèle ce qui, dans toute règle, tient des modalités collectives et sociales de sa définition autant que de son application subjective, même s'il semble arbitraire de l'exclure par principe de tout ensemble social, de toute institution. D'ailleurs, l'institution institue le flou par le déni du caractère arbitraire de la règle. L'opération de déni de cet arbitraire social, producteur de discriminations scolaires mais aussi de marges d'interprétations, sert d'opérateur de rationalisation des règles relayées par leur négociabilité: ce que nous désignons du vocable de plurivocité des règles. Aucune institution, aussi régulée soit-elle, ne peut fonctionner sans laisser des marges de liberté, d'improvisation, d'interprétation, de négociation à ses acteurs. Ce que la féminisation du corps enseignant tend d'ailleurs à rendre plus visible dans les stratégies féminines d'imposition pédagogique. Mais dans la machinerie sociale que constitue l'organisation scolaire, si les règles sont négociables, elles sont présentées comme des nécessités - et elles le sont -, en tant qu'elles sont et qu'elles sont ce qu'elles sont. La spécificité de l'école, comme lieu et comme moment de la règle, ne tient pas à la définition de cet arbitraire collectif, qui est le propre de toute institution, mais au paradoxe d'un système qui, produisant un arbitraire imposé quoique discuté, le présente comme rationalité intangible. La neutralité de la décision, à l'instar de celle de la laïcité, est collectivement revendiquée. La façon dont les enseignants vont jouer leur rôle, définir la situation en classe, donner à voir leur présentation de soi-depuis les premières rencontres avec la classe, et tout au long de leurs interactions - va préserver l'ordre scolaire ou engendrer, au contraire, des formes de déviance banales ordinaires ou des formes plus critiques (anomiques), c'est-à-dire des ruptures dans les définitions des situations mises à jour par Boumard (2000). L'apparente adhésion collective à cet ordre scolaire, localement réinterprété dans chaque situation de classe, achoppe sur les conflits d'intérêts et de position de statut et de reconnaissance sociale. Cela d'autant que lieu, moment et interaction participent de la définition de cet ordre scolaire, jamais totalement différent, jamais totalement identique. Il s'ensuit un processus 
d'interactions producteur de violences, symboliques ou physiques, engendrées par des divergences d'interprétation des règles dans une société où le sujet est moins assujetti. Nombreux sont les exemples de réinterprétations quotidiennes des règles scolaires au gré des individus, des situations, des établissements scolaires, des interactions: de l'absentéisme (chaque enseignant à l'aune de sa relation à l'élève, à la classe et à sa propre histoire d'élève, mais aussi à l'institution) aux normes de ponctualité (dans les retards acceptables ou non, en matière de gestion des horaires ou de rendus de devoirs, etc.); des propos admissibles dans l'enceinte de la classe (possibilité ou pas d'interrompre l'enseignant, langage soutenu ou vulgaire, etc.) aux modalités d'évaluation des performances des élèves (nombre de notes, détermination des coefficients, rapports à la note au sens des travaux critiques de la docimologie, etc.); aux décisions d'orientation des conseils de classe et aux évaluations des enseignants dans le cadre des inspections. Il est patent de constater notamment que les décisions d'orientation des élèves au sein des conseils de classe sont le résultat de rationalités plurielles portées par des acteurs statutairement distingués (chef d'établissement, professeur principal, matière enseignée, grade de l'enseignant, sexe et âge de l'enseignant, mais aussi parents d'élèves, conseiller d'orientation et délégués des élèves). Ces rationalités répondent à la nécessaire gestion des flux d'élèves, à la hiérarchie sociale des disciplines, à la mise en œuvre délocalisée des choix de politique publique, aux intérêts catégoriels, aux rapports personnels de pouvoir, aux modes de présentation de soi, aux stratégies d'imposition du chef d'établissement, aux solidarités de corps ou aux injonctions syndicales. Reposant sur des performances, elles-mêmes évaluées selon des modalités sociales autant que disciplinaires, la décision d'orientation qui s'impose à l'élève (même s'il dispose de modalités de contestation de la décision, elles-mêmes encadrées de règles plurivoques) est pensée par l'ensemble des acteurs qui ont intériorisé les règles du jeu scolaire (centraux autant que périphériques), comme des décisions neutres et impersonnelles, quoique destinées à une personne particulière.

\subsection{La laïcité et le déni du politique}

Or, la laïcité est bien cette idéologie de neutralisation des différences par le lieu scolaire, cette revendication de neutralité politique (pas de politique à l'école), cette négation de l'altérité constitutive des publics scolaires, d'ailleurs de plus en plus hétérogènes, cette violence symbolique. Parce que la laïcité, en tant que norme centrale de cohésion de l'institution scolaire, sert de socle idéologique pour faire tenir ensemble ce qui est désuni, le caractère violentogène de toute stratégie d'imposition pédagogique, donc de toute institution scolaire et de tout ordre social, ne peut qu'être un impensé radical de l'institution. Dans la machinerie sociale que constitue une organisation scolaire, les règles se négocient et, en même temps, elles sont présentées comme des indiscutables. Ce qui est à noter, ce n'est pas la définition de cet arbitraire collectif, qui est le propre de toute organisation, mais bien le paradoxe, aporie du système qui, produisant un arbitraire imposé, quoique discuté, 
le présente comme rationalité intangible. Les pratiques scolaires s'organisent donc entre négociation/conflits/interprétations. La difficulté centrale, sur ce sujet, c'est que toutes les pratiques scolaires sont imprégnées de ce processus de négociation/ conflits/interprétation. Ainsi, pour saisir ce qui se joue dans la production de l'excellence scolaire et, par là, de certaines formes de déviances, il faut comprendre que, comme dans toute organisation, les règles sont non seulement collectivement interprétées, mais singulièrement réappropriées par les sujets. Par delà la plurivocité structurelle de toute règle, les stratégies d'imposition scolaire, en niant l'arbitraire de la règle, gomment la question du pouvoir par la neutralisation des processus sociaux et politiques qui déterminent toute décision. Toutefois, l'institution scolaire se construit autour d'une représentation collective largement intériorisée par ses acteurs. Elle se présente comme une organisation rationnelle: chacun croit participer à une mise en ordre univoque, alors même que l'ordre est pluriel et mouvant. La puissance souterraine du social est le moteur d'un ordre dialectique de conflits, consensus et négociations, par les réinterprétations plurielles des règles, les négociations et jeux de pouvoir, les logiques d'action plurielles qui président à la continuelle définition/redéfinition des modes de décision. Ainsi, notre argumentaire vise ici, non à dénoncer le caractère résolument politique de l'institution scolaire, mais à l'affirmer comme nécessité indépassable. Certes, la nature du lien politique présente une forme d'ambiguité, puisqu'elle recouvre à la fois une dimension horizontale de vouloir-vivre ensemble, de dialogue (ce qui rejoint l'idée d'un espace de non-guerre où les individus partagent un nous qui leur est commun) et une dimension verticale faite de rapports conflictuels, de domination et d'exclusion. Or, l'institution scolaire, à l'image du politique, est soumise à un certain nombre de contradictions. En tant que lieu du politique, elle doit, par exemple, affronter la tension qui oppose des principes irréductibles: se présenter comme la raison en marche dans le droit fil de sa lignée historique émancipatrice, tout en assumant désormais son caractère pluriel. C'est bien dans ce caractère paradoxal que l'école joue sa pérennité en tant qu'agent d'intériorisation des règles collectives. Si les règles ne sont jamais univoques, cet arbitraire collectif définit un espace de possibles et donc, par ricochet, c'est bien ce rapport à la règle qui fixe l'idée même de déviances (au sens d'écart durable par rapport aux normes socialement établies).

Au cœur de l'institution scolaire, la violence de l'institution est bien là, non seulement en ce qu'elle impose des limites à la jouissance, mais en ce qu'elle dénie sa genèse sociale et son fonctionnement politique. S'il y a déni du politique à l'école, c'est que la construction conflictuelle/consensuelle d'un ordre scolaire temporairement fixé au moyen d'une architectonique de relations de pouvoirs (par exemple le fait de penser que la note est une valeur objective de la performance scolaire) est niée comme processus politique, car pensée comme expression de la raison. Ce déni collectif s'exprime sous de nombreuses expressions: déni de la gestion des corps individuels réduite à celle d'un corps politique abstrait - la classe -, ou encore 
contrôle des esprits, au moyen de la production simplifiante/mutilante d'une mémoire collective sélective et propre à narrer l'Histoire légitime de l'ordre national, républicain et moniste. Il relève d'un travail de scotomisation du Politique, en ce sens que le caractère plurivoque, interprétatif et négociable des règles scolaires est généralement l'impensé du lieu (l'école) et du milieu (les enseignants en tant que porteurs de l'institution). Rappelons que la scotomisation est un processus de dénégation qui permet de ne pas voir des contenus, images, souvenirs trop angoissants. Il y a constitution d'un véritable scotome psychique sélectif, rétrécissant le champ de conscience et produisant une amnésie collective.

\section{Conclusion}

Cette empreinte indélébile du politique dans le lieu scolaire, que l'institution cache (et se cache à elle-même), manifeste une contradiction fondamentale du système: l'imposition de règles motrices de la vie au sein de l'organisation scolaire doit légitimer cet arbitraire collectif autour d'une imputation commune qui en rationalise au moins apparemment le fonctionnement. Hier, cette imputation commune allait socialement de soi; aujourd'hui, elle est à reconstruire en dialogue avec l'émergence nouvelle du sujet, nouveau paradigme du social autant que de la sociologie. La question de la nécessaire intériorisation de la loi scolaire gomme celle de l'arbitraire des règles. Il est probable que la violence symbolique de l'institution s'exprime dans son impossibilité à penser et à traduire, dans des espaces de médiation, l'arbitraire originel/original de toute règle. Les sociologies naissantes du sujet peuvent éclairer cette tension entre le collectif et l'individuel, non tant sur le mode de l'emprise du social sur l'individuel ou de la production du singulier par le collectif, mais comme une analyse de la production sociale et scientifique des modalités de dialogues entre raison sociale et sujet individuel postmoderne.

\section{Notes}

1. En référence aux travaux de Foucault (1975).

2. L'herméneutique désigne le fait d'interpréter autant le texte que l'événement qui en est l'origine.

3. En lien avec le panoptique de Bentham (1791), repris par Foucault (1975), au sens de lieu d'où la possibilité du regard de l'autre est toujours possible et jamais certaine.

4. Qui a trait aux fins de l'homme et en assigne les buts.

5. Ce néologisme est proposé pour rendre compte des regroupements culturels et cultuels actuels, très distincts des anciennes paroisses. Il s'agit d'identification au groupe communautaire culturel ou cultuel sans partage de l'ensemble des dogmes du groupe de référence. 
ENGLISH TITLE - The concept of subject from «foucaldian» to post-modern views: laicism and denial of policies in the French educational system

SUMMARY - When modern societies become post-modern societies, the concept of subject as well as the construction of school rules require a re-evaluation. If within the school setting control becomes regulatory, the matter of arbitrariness and plurivocality concerning rules is erased by a necessary interiorization of school law. The specificity of the French educational system lies in the historical republican creation of the secularity of schools, which is much too often mistaken for political or religious neutrality. Denying a possible negotiability and interpretation of academic standards confirms the political nature of the educational system and shows how difficult it is to reflect in a collective way regarding the relationship/place of the school.

KEY WORDS • school, laicism, rules and norms, political power, representations.

TíTULO EN ESPAÑOL • Del sujeto foucaultino al sujeto posmoderno. Laicidad y negación del carácter político en el sistema escolar francés

RESUMEN - El paso de las sociedades modernas a las sociedades posmodernas lleva a la reevaluación de la noción de sujeto y de las modalidades de construcción de la norma escolar. En la escuela, si el control llega a ser regulación, la cuestión de la necesaria interiorización de la ley escolar borra la del arbitrario y de la multivocidad de las reglas. La especificidad del sistema educativo francés procede de la construcción histórica republicana de la laicidad, abusivamente comprendida como neutralidad política o religiosa. La negación del carácter negociable e interpretativo de las normas escolares asegura el carácter político de la institución escolar y las dificultades para pensar colectivamente el vínculo/espacio escolar.

PALABRAS CLAVES • escuela, laicidad, reglas y normas, poder político, representaciones.

\section{Références}

Ardoino, J. (1985). Retrouver le politique. Dossier: l'acteur et l'institution. Cahiers pédagogiques, 234.

Boumard, P. (2000). L'École, les jeunes, la déviance. Paris, France: Presses universitaires de France.

Citron, S. (1989). Le mythe national. L'histoire de France en question. Paris, France: Les Éditions ouvrières.

Coq, G. (1995). Lä̈cité et République. Le lien nécessaire. Paris, France: Belin.

De Munck, J. (1997). Du souci de soi contemporain. Dans G. Bajoit et E. Belin (Dir.): Contributions à une sociologie du sujet. Paris, France: L'Harmattan.

Durkheim, É. (1967). De la division du travail social (8 édition). Paris, France: Presses universitaires de France.

Elias, N. (2002). La civilisation des mours. Paris, France: Calmann-Lévy.

Étienne, B., Giordan, H. et Lafont, R. (1999). Le temps du pluriel. La France dans l'Europe multiculturelle. La Tour d'Aigues, France: Éditions de l'Aube.

Foucault, M. (1975). Surveiller et punir. Paris, France: Gallimard. 
Gautherin, J. (2000). L'universalisme de l'École laïque à l'épreuve. Dans A. Van Zanten (Dir.): L'École, état des savoirs. Paris, France: Armand Colin.

Giddens, A. (2005). La constitution de la société. Paris, France: Presses universitaires de France.

Haby, R. (1975). Pour une modernisation du système éducatif. La Documentation française, $\mathrm{n}^{\circ}$ spécial.

Mabilon-Bonfils, B. et Saadoun, L. (2001). Sociologie politique de l'École. Paris, France: Presses universitaires de France.

Maffesoli, M. (2002) La transfiguration du politique. Paris, France: Table Ronde.

Sen, A. K. (1993). Éthique et économie, et autres essais. Paris, France: Presses universitaires de France.

Weber, E. (1983). La fin des terroirs. Paris, France: Fayard.

\section{Correspondance}

Beatrice.mabilon-bonfils@wanadoo.fr

Cet article a été révisé par Christelle Lison.

Texte reçu le: 25 janvier 2006

Version finale reçue le: 27 juillet 2007

Accepté le: 15 août 2007 